\title{
Localization of gauge fields and monopole tunnelling
}

\author{
G. Dvali, ${ }^{1,2}$ H. B. Nielsen, ${ }^{1,3}$ and N. Tetradis ${ }^{4}$ \\ ${ }^{1}$ CERN, Theory Division, CH-1211 Geneva 23, Switzerland \\ ${ }^{2}$ Center for Cosmology and Particle Physics, Department of Physics, New York University, New York, New York 10003, USA \\ ${ }^{3}$ The Niels Bohr Institute, Copenhagen DK 2100, Denmark \\ ${ }^{4}$ Department of Physics, University of Athens, Zographou 157 84, Athens, Greece
}

(Received 22 November 2007; published 7 April 2008)

\begin{abstract}
We study the dynamical localization of a massless gauge field on a lower-dimensional surface (2-brane). In flat space, the necessary and sufficient condition for this phenomenon is the existence of confinement in the bulk. The resulting configuration is equivalent to a dual Josephson junction. This duality leads to an interesting puzzle, as it implies that a localized massless theory, even in the Abelian case, must become confining at exponentially large distances. Through the use of topological arguments we clarify the physics behind this large-distance confinement and identify the instantons of the brane world-volume theory that are responsible for its appearance. We show that they correspond to the (condensed) bulk magnetic charges (monopoles), that occasionally tunnel through the brane and induce weak confinement of the brane theory. We consider the possible generalization of this effect to higher dimensions and discuss phenomenological bounds on the confinement of electric charges at exponentially large distances within our Universe.
\end{abstract}

DOI: 10.1103/PhysRevD.77.085005

PACS numbers: 11.15. $-\mathrm{q}, 11.25 .-\mathrm{w}, 11.27 .+\mathrm{d}$

\section{INTRODUCTION}

The dynamical localization of massless modes is a very interesting physical phenomenon, that goes against the naive quantum-mechanical intuition according to which a bound state naturally has a mass of the order of the inverse localization width. The exceptions from this "rule" are well known for spin- 0 and spin- $1 / 2$ systems. The massless spin-0 scalars can be localized on lower-dimensional solitons as Goldstone bosons (sound waves) of the broken translational invariance. The appearance of the fermionic zero modes in the field of topological defects is due to topologically nontrivial boundary conditions for the fermion mass and is guaranteed by the index theorem [1].

In contrast, no analogue of the Goldstone or the index theorems exists for the dynamical localization of massless spin-1 fields. In string theory the objects that support massless spin-1 excitations on their world-volume are $D$-branes [2]. The massless gauge fields are excitations of the open strings that end on the brane. Therefore, they are intrinsically lower dimensional "from the beginning." In other words, it is unclear how to trace their higherdimensional counterparts. In the present work we shall be concerned with the dynamical localization of gauge fields within field theory.

It was argued in [3] that, at least on asymptotically flat spaces, the necessary and sufficient condition for the localization of massless gauge fields on a lower-dimensional surface, embedded in a higher-dimensional bulk space, is bulk confinement. That is, at high energies the localized gauge theory must become part of a confining higherdimensional theory. As shown in [3], a simple $(3+1) \rightarrow$ $(2+1)$ model illustrating such a dynamical localization of a massless photon is a $(3+1)$-dimensional $S U(2)$ gauge theory Higgsed down to $U(1)$ on a $(2+1)$-dimensional wall (brane). The dynamical localization of a (perturbatively) massless photon follows from the fact that the $U(1)$ gauge field becomes part of a confining gauge theory in the bulk, with a mass gap $\sim \Lambda$. A localized photon can only escape into the bulk in the form of a massive glueball. Thus, the bulk confinement creates a barrier that confines the photon to the brane. The localized photon is perturbatively massless because the $U(1)$ gauge symmetry is never Higgsed.

The immediate consequence of this picture is the existence of electric flux tubes that end on the brane. The close resemblance between these and the open strings of the $D$-brane theory is rather striking. It poses the question whether the connection between the open strings and the massless gauge fields has a common underlying origin both in string and field theory [4]. This analogy was also discussed earlier in the context of the connection between the domain walls of supersymmetric gauge theories and $D$-branes [5].

In the present work, we shall be concerned with phenomena that arise when one tries to think about the localization mechanism of [3] as the creation of a dual Josephson junction [6]. Indeed, the appearance of confinement is believed to be equivalent to the condensation of magnetic charges [7]. The brane then becomes a dual insulator, sandwiched between two infinite magnetic superconductors. For the ordinary Josephson junction, it is well known that the Meissner effect (the confinement of magnetic charges) penetrates the layer at the quantummechanical level, because of the presence of tunnelling Josephson currents [8]. Hence, even on the layer the magnetic charges are not in a truly Coulomb phase, but get confined at large distances. Because of the tunnelling nature of the effect, the magnetic confinement scale of the $(2+1)$-dimensional theory is exponentially sup- 
pressed by the width of the layer. Nevertheless, this is a real effect that has been observed. In complete analogy with the standard Josephson junction, the presence of tunnelling magnetic currents in the dual picture is expected to induce exponentially weak confinement of electric charges located on the brane. We emphasize that the appearance of a very small mass gap through confinement should not be confused with the presence of a mass term of the Higgs or Proca type for the photon. Perturbatively the photon is massless, and only at exponentially large distances confinement sets in.

While trying to apply this reasoning to the $S U(2)$ model of [3] one encounters a puzzle. As suggested in [6], if duality works the $(2+1)$-dimensional $U(1)$ theory must become confining at exponentially large distances. On the other hand, one may wonder how this is possible, since at distances above the brane width an effective $(2+1)$ dimensional theory is just a pure perturbatively massless $U(1)$. Where is the confining dynamics coming from?

In the present work we shall resolve the above puzzle. We shall argue that the confinement of the effective $(2+1)$-dimensional theory on the brane can be understood in terms of the confining dynamics of a truly $(2+1)$ dimensional theory with compact $U(1)$. The latter was first studied by Polyakov [9]. Perturbatively, the theory seems to be in the Coulomb phase. However, Polyakov demonstrated that the IR dynamics is governed by instantons, whose presence results in the confinement of electric charges.

The identification of the two low-energy theories requires some care. In order to achieve it we employ a topological method, in which we view a $D$-dimensional theory as a slice of a $(D+1)$-dimensional one. This method allows us to classify the instantons of the $D$-dimensional theory as the monopoles of the $(D+1)$ dimensional one that tunnel across the slice. Applying this method to the model of [3], we show that the instantons are the monopoles that tunnel through the brane. These instantons lead to the confinement of electric charges at exponentially large scales, in agreement with the conclusions of [6].

We shall also discuss briefly the relevance of this phenomenon for higher-dimensional cases, and possible phenomenological implications of the large-distance electromagnetic confinement. We point out that the existence of the galactic magnetic field already puts a very severe restriction on the confinement scale. This is different to the situation for a photon mass of the Higgs or Proca type, which essentially is not restricted by the galactic magnetic field [10].

\section{LOCALIZATION OF GAUGE FIELDS}

\section{A. Problems with localization by the bulk Higgs effect}

If the bulk mass that confines the photon to a lowerdimensional subsurface or layer ("brane") is of the Higgs or Proca type, the localization does not produce a massless electric field in the effective lower-dimensional theory. The localized electric photon acquires a large mass because of the same bulk Higgs effect that confines it to the brane. As the gauge field is in the Higgs phase in the bulk, electric charge screening also penetrates the brane resulting in an exponentially decaying electric field. The problem can be illustrated by the following simple model that localizes the photon onto a $(2+1)$-dimensional surface. Consider an Abelian Higgs model and assume a potential for the Higgs field with two degenerate minima: one located at $\Phi=0$ and the other at $|\Phi|=M \neq 0$. In the $\Phi=0$ vacuum the photon is massless and the $U(1)$-theory is in the Coulomb phase. The test charges create a long-range $1 / r$ potential. In the $\Phi \neq 0$ vacuum the photon has a mass $m_{\gamma} \sim M$ and the theory is in the Higgs phase. In this vacuum the test charges are screened and generate a Yukawa-type potential $\sim \exp \left(-m_{\gamma} r\right) / r$.

The two phases can coexist. In such a case they will be separated by domain walls of tension $\sim M^{3}$ and width $\sim M^{-1}$. Throughout the wall the expectation value of $|\Phi|$ smoothly interpolates between 0 to $M$ over a distance $\sim M^{-1}$. One simple choice of $V(\Phi)$ that realizes this picture is

$$
V(\Phi)=\left(|\Phi|^{2}-M^{2}\right)^{2} \frac{|\Phi|^{2}}{M^{2}} .
$$

(For simplicity we have set all the dimensionless coupling constants equal to one.) This theory has a single mass scale $M$ and vacua at $\Phi=0$ and $|\Phi|=M$. We have chosen the parameters in such a way that the vacua are degenerate. This choice allows for the two phases to coexist, with a static wall in between. Of course, many other choices of $V(\Phi)$ with similar properties are possible [4].

Consider a situation in which a layer of the would-be Coulomb vacuum with $\Phi=0$ is "trapped" between two $|\Phi|=M$ phases. This can be achieved by placing a wall and an antiwall parallel to each other at a certain distance $d$. If $z$ is the coordinate perpendicular to the walls, $|\Phi|$ would vary from $M$ to 0 and back to $M$, as one crosses the wall and antiwall system from $z=-\infty$ to $z=+\infty$. As we said above, the width of the walls that bound the $\Phi=0$ vacuum is $\sim M^{-1}$. Of course, this system is not really stable, and not even static, but if $d \gg M^{-1}$ the force between the wall and antiwall is exponentially suppressed by a factor $\exp (-M d)$, so that the system can be considered to be static over the time scales of interest.

Naively, one may think that this setup localizes a massless photon in an effective $(2+1)$-dimensional theory on the layer, because $\Phi=0$ there. However, this is not the case because the would-be Coulomb vacuum is sandwiched between two Higgs phases and the charge screening penetrates there. Formally, the absence of the massless mode can be seen directly from the mode expansion analysis, but the physical reason is clear: The two Higgs phases 


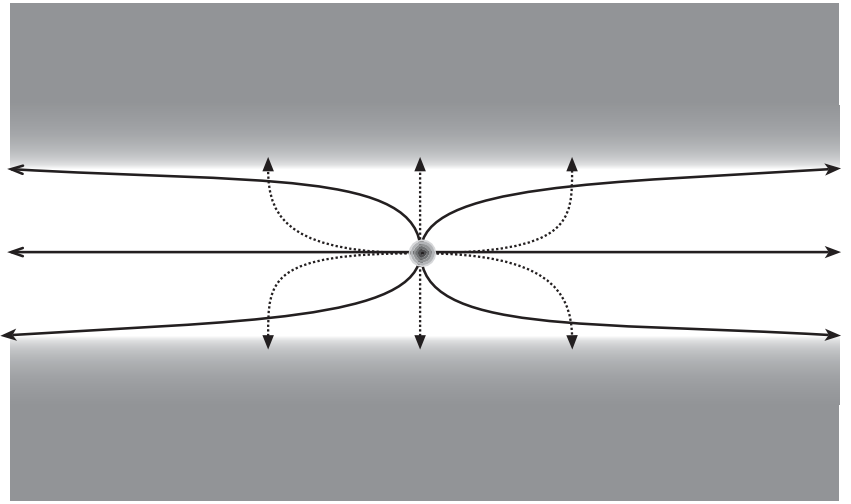

FIG. 1. Electric (dotted) and magnetic (solid) field lines generated by a fictitious dyon (electrically and magnetically charged particle) in the Josephson junction. In the dual picture the roles of the electric and magnetic fields are reversed.

bounding the would-be Coulomb vacuum are superconductors, with a lot of free charges available in the vacuum. Any test charge placed in the layer polarizes this vacuum and creates image charges that screen it. In the language of electric flux lines, the fact that the bulk is superconducting implies that the electric flux lines of the $(2+1)$ dimensional source can end on the boundary in the vicinity of the image charges (see Fig. 1). Hence, there is no flux conservation in the $(2+1)$-dimensional theory. The theory is in the Higgs phase, both on the layer and in the bulk, with comparable masses for the photon.

\section{B. Localization by the dual Higgs effect}

The lesson from the previous analysis is that, in order to localize a massless photon, the electric flux lines should not be able to either spread out of the brane or end on its boundary. In other words, the bulk medium must repel the electric flux lines, without breaking or terminating them on any image charges. This will induce flux conservation within the $(2+1)$-dimensional theory on the layer, and, by Gauss's law, the $(2+1)$-dimensional Coulomb phase. This means that the bulk condensate that repels the flux lines must be of the magnetic type. In such a case the electric charges in the bulk will be confined, but not screened.

This can be achieved if the Abelian $U(1)$ symmetry becomes part of a confining theory at a certain scale $\Lambda$. The simple model (a variation of the original one) that realizes this consists of an $S U(2)$ gauge theory with a single adjoint Higgs $\Phi^{a}(a=1,2,3)$. The potential is a non-Abelian generalization of (1):

$$
V(\Phi)=\left(\Phi^{a} \Phi^{a}-M^{2}\right)^{2} \frac{\Phi^{a} \Phi^{a}}{M^{2}} .
$$

Again, this potential has the two vacua, $\Phi^{a}=0$ and $\Phi^{a}=$
$M n^{a}$ (where $n^{a}$ is an arbitrary unit vector in threedimensional space). In the first vacuum, the theory is in the confining phase and there is a mass gap $\sim \Lambda$. All the states in this vacuum are massive glueballs. In the second vacuum, the theory is Higgsed down to $U(1)$ and there is a massless photon. Perturbatively, test charges in this vacuum are in the Coulomb phase. As in the previous example, the two phases may coexist, separated by domain walls of tension $\sim M^{3}$ and width $\sim M^{-1}$.

Let us now consider a layer of the Abelian $U(1)$ vacuum sandwiched between the two $S U(2)$ confining phases. The width of the layer (distance between the wall and antiwall) is $d$. We shall assume that $M \gg d^{-1}, \Lambda$. Again, the walls can be considered as being static in the time scales of interest. In the layer of the $U(1)$ vacuum all the states except the photon are massive. Although the photon has no Higgs or Proca mass anywhere, it is repelled out of the bulk by the confining dynamics. It cannot enter the bulk without becoming a massive glueball. In other words, the electric flux of a test source can only penetrate the bulk in the form of an $S U$ (2) flux tube, with a tension $\sim \Lambda^{2}$. As a result, perturbatively the effective low-energy theory below the scale $d^{-1}$ is a $(2+1)$-dimensional theory of a massless photon. Since below such energies all the heavy states decouple, and there are no light charges available, one expects the photon to remain in the Coulomb phase down to arbitrarily low energies. But is this true?

\section{The puzzle}

As far as the low-energy theory is concerned, the bulk confinement picture is dual to the bulk Higgs model. In the $U(1)$ Higgs phase, test magnetic monopoles are confined because they are connected by the Nielsen-Olesen magnetic flux tubes [11]. These flux tubes are dual to the ones that confine test charges in the unbroken $S U(2)$ phase. If duality holds, and the bulk confinement picture indeed creates an electric Coulomb phase on the layer, the bulk Higgs theory should create a magnetic Coulomb phase there.

This analogy is precisely the source of the puzzle. The crucial point is that in the bulk Higgs picture the bulk is a superconductor, and there must be a Josephson effect. Because of the phase difference of the condensates on the two sides of the layer, charges can tunnel through it and create a current. As a result, the magnetic charges on the layer cannot remain in the Coulomb phase for arbitrarily large distances, but only up to an exponentially large separation, after which they get confined. In other words, the flux of the Nielsen-Olesen tube can only spread out to an exponentially large distance but not to infinity. This fact poses the question of whether a dual analogue of the Josephson effect takes place in the bulk confinement model, and whether the electric charges on the layer remain in the Coulomb phase or become confined at exponentially large distances. 


\section{THE PHYSICS OF THE JOSEPHSON JUNCTION}

In this section we summarize the physics of the Josephson junction and discuss the implications for the dual picture in which there is a condensate of magnetic charge in the bulk. Our arguments are quite general and do not rely on the details of the theory in which electric and magnetic charges are incorporated consistently.

\section{A. The Josephson junction}

Let us consider the Abelian Higgs model in $3+1$ dimensions, described by the action

$$
S=\int d^{4} x\left\{-\frac{1}{4} F_{\mu \nu} F^{\mu \nu}+\left|\left(\partial_{\mu}+i g A_{\mu}\right) \Phi\right|^{2}-V(\Phi)\right\},
$$

with $F_{\mu \nu}=\partial_{\mu} A_{\nu}-\partial_{\nu} A_{\mu}$. In a simple configuration that could localize the gauge field on a brane, the scalar field $\Phi$ has a zero expectation value inside the brane and a constant nonzero value in the bulk. For our discussion we shall assume that $\Phi=0$ for $|z|<d / 2$ and $\Phi=\rho \exp (i \omega)$ for $|z| \geq d / 2$. As a result, the gauge field becomes massive in the bulk through the Higgs mechanism, while it remains massless inside the brane.

The Abelian Higgs model in the spontaneously broken phase is equivalent to the Ginzburg-Landau theory of superconductivity. A well-known property of superconductors is the existence of frictionless currents that can flow without a potential. For the tree-level Lagrangian of Eq. (3) the current density is given by

$$
J_{\text {tr }}^{\mu}=-2 g \rho^{2} \partial^{\mu} \omega-2 g^{2} \rho^{2} A^{\mu} .
$$

The phase $\omega$ is unobservable for a bulk superconductor, as it can be eliminated through a gauge transformation. The superconducting currents flow near the surface of the material and expel magnetic fields from it. This property, the Meissner effect, can be understood also as a consequence of the photon mass $\sqrt{2} g \rho$. The magnetic field decays over a distance $\lambda \sim(g \rho)^{-1}$. Deep inside the superconductor, the gauge field is zero and the current of Eq. (4) vanishes.

The resulting configuration (Fig. 1) corresponds to a Josephson junction: two superconducting regions separated by a thin layer of nonsuperconducting material. The phase $\omega$ cannot be eliminated completely in this case. More specifically, we can define the gauge-independent phase difference between two points [8]

$$
\Delta \omega_{P_{1} P_{2}}=\omega\left(P_{2}\right)-\omega\left(P_{1}\right)-g \int_{P_{1}}^{P_{2}} \vec{A} \cdot d \vec{l} .
$$

The gauge-dependent phases $\omega\left(P_{1}\right)$ and $\omega\left(P_{2}\right)$ can be eliminated for convenience through an appropriate gauge transformation. The phase difference $\Delta \omega$ between two points on either side of the junction can be nonzero [8].
As a result of the presence of a phase difference, a superconducting current, the Josephson current, flows across a junction. This can be shown on general grounds [12] by noticing that, beyond tree level, the effective action of the system depends on $\Delta \omega$ through quantum effects (tunnelling of charges across the junction). The current can be obtained from the effective matter action through differentiation with respect to the gauge field. The dependence of the action on a phase difference given by Eq. (5) immediately leads to the presence of a current. This tunnelling current does not require a potential difference between the two superconducting regions in order to flow.

We can change each of the phases $\omega\left(P_{1}\right), \omega\left(P_{2}\right)$ by a multiple of $2 \pi$ without altering its physical significance. This means that the effective action and the Josephson current must be periodic functions of $\Delta \omega$. We parametrize the current density as $[8,12]$

$$
J^{3}(\Delta \omega)=J_{\max }^{3} \sin (\Delta \omega),
$$

where $J_{\max }^{3}$ is its maximum value. We emphasize that $J^{3}$ is a tunnelling current. [The classical current of Eq. (4) is zero in the brane.] The maximum value $J_{\max }^{3}$ can be taken as a phenomenological parameter that can be very small in units of the typical mass scale of the potential $V(\phi)$ in Eq. (3).

We can now consider the behavior of the electromagnetic field inside the brane. We employ the Maxwell equations in the presence of an external current density given by Eq. (6). They correspond to the equations of motion derived from the tree-level Lagrangian of Eq. (3) with $\phi=0$ and an external current. The presence of the bulk superconductors imposes certain conditions on the solutions of these equations. The electric field parallel to a conductor is zero near its surface. As we discussed in the previous section based on the arguments of [3], this means that electric field lines must end perpendicularly to the boundary of the Josephson junction (Fig. 1). For a point charge, the electric field dies off within a distance $\sim d$ in the $x$, $y$-directions. As we are interested in the low-energy behavior of the system, we do not consider configurations with variations of the fields at short distances. This means that we can approximate $E_{x}=-\partial A^{0} / \partial x-\partial A^{1} / \partial t$ and $E_{y}=-\partial A^{0} / \partial y-\partial A^{2} / \partial t$ as zero and assume that $E_{z}=$ $-\partial A^{3} / \partial t$ is independent of $z$ inside the brane. The magnetic field has a continuous $z$-component at the surface of a conductor. As it is zero inside the superconductor, we assume that $B_{z}=-\partial A^{1} / \partial y+\partial A^{2} / \partial x$ vanishes everywhere. The components $B_{x}=\partial A^{3} / \partial y, B_{y}=-\partial A^{3} / \partial x$ are nonzero in the brane and vanish exponentially within a distance $\lambda \sim(g \rho)^{-1}$ in the bulk. The magnetic field lines are localized inside the brane (Fig. 1).

It is clear that the only unconstrained component of the gauge field is $A^{3}$, whose value is related to the gaugeinvariant phase difference $\Delta \omega$ across the brane. From Eq. (5) with $\omega=0$ we obtain 


$$
\Delta \omega=-g \int_{P_{1}}^{P_{2}} \vec{A} \cdot d \vec{l} \simeq \Delta \omega_{P_{1} P_{2}}=-g d A^{3}(P) .
$$

Here $P_{1}, P_{2}$ are points opposite each other on either side of the brane and $A^{3}(P)$ the value of the gauge field inside the brane. The equation of motion of $\Delta \omega$ can be derived through an elementary use of Maxwell's equations [6,8]. It is

$$
\partial^{i} \partial_{i}(\Delta \omega)+g d J_{\max }^{3} \sin (\Delta \omega)=0,
$$

where $i=0,1,2$. We conclude that there is one light mode on the brane that obeys the sine-Gordon equation. This mode corresponds to the third component of the gauge field or the phase difference between the condensates on either side of the brane: $\Delta \omega=-g d A^{3}$. If we consider weak fields $(\Delta \omega \ll 1)$, we can approximate Eq. (8) as

$$
\left[\partial^{i} \partial_{i}+m^{2}\right] \Delta \omega=0,
$$

with $m^{2}=g d J_{\max }^{3}$. In realistic Josephson junctions Eq. (9) implies the presence of a Meissner effect even in the nonsuperconducting material [8]. Applied electromagnetic fields decay over a distance $\sim\left(g d J_{\max }^{3}\right)^{-1 / 2}$. This phenomenon has been observed experimentally. The decay length in the junction can be orders of magnitude larger than the decay length in the superconductor. Also solitonic configurations can appear, corresponding to solutions of Eq. (8) [8].

We can conclude that the effective $(2+1)$-dimensional theory includes only one light physical degree of freedom $\left(A^{3}\right.$ or $\Delta \omega$ ). The nonvanishing components of the electromagnetic field are

$$
B_{x}=\frac{\partial A^{3}}{\partial y} B_{y}=-\frac{\partial A^{3}}{\partial x} E_{z}=-\frac{\partial A^{3}}{\partial t},
$$

where we have assumed no $z$-dependence. However, we would like to have nonvanishing $E_{x}, E_{y}, B_{z}$ in order to generate an effective $(2+1)$-dimensional theory of electric charges. A possible remedy for this situation is provided by the suggestion of Ref. [3]. The material in the bulk must be a dual superconductor [7]. In other words, there must be a condensate of magnetic charge in the bulk.

\section{B. The dual Josephson junction}

It is believed that dual superconductivity is realized in the confining phase of gauge theories. The particular implementation of Ref. [3] employs an $S U$ (2) gauge theory coupled to a scalar field in the adjoint representation (the Georgi-Glashow model) [13]. Inside the brane the $S U(2)$ symmetry is broken down to $U(1)$ through a nonzero expectation value of the scalar field. The low-energy theory is in the Coulomb phase and a massless photon should emerge. In the bulk the scalar field has a zero expectation value and the theory is in the confining phase. All excitations are very massive, and this prevents the photon that is localized on the brane from entering the bulk.
In our discussion here we shall use only the main elements of the above picture. We consider electromagnetism in the presence of $U(1)$ magnetic charge. We assume that a magnetic condensate forms in the bulk, with the appearance of frictionless currents. In the absence of electric charge, we can use a phenomenological description

$$
S=\int d^{4} x\left\{-\frac{1}{4} \tilde{F}_{\mu \nu} \tilde{F}^{\mu \nu}+\left|\left(\partial_{\mu}+i g_{m} C_{\mu}\right) \psi\right|^{2}-V(\psi)\right\} .
$$

The dual gauge field $C^{\mu}$ is defined through the duality $[14,15]$

$$
F^{\mu \nu} \rightarrow \tilde{F}^{\mu \nu}=\frac{1}{2} \epsilon^{\mu \nu \lambda \sigma} F_{\lambda \sigma}=\partial^{\mu} C^{\nu}-\partial^{\nu} C^{\mu},
$$

and $g_{m}$ is the magnetic charge. We emphasize at this point that, in the presence of a magnetic current, the field $F^{\mu \nu}$ does not have a simple description in terms of a gauge field $A^{\mu}$ [15]. Only the dual field $\tilde{F}^{\mu \nu}$ can be expressed simply through $C^{\mu}$. A frictionless magnetic current flows near the surface of the regions of nonzero expectation value for the magnetic condensate $\psi=\sigma \exp (i \chi)$. At tree level it is given by

$$
\tilde{J}_{t r}^{\mu}=-2 g_{m} \sigma^{2} \partial^{\mu} \chi-2 g_{m}^{2} \sigma^{2} C^{\mu}
$$

A dual Meissner effect prevents the electric field from entering the regions with $\psi \neq 0$.

The system of Fig. 1 can be viewed now as a dual Josephson junction with a tunnelling magnetic current $\tilde{J}^{3}$ flowing across the brane. It is clear from Eq. (13) that the gauge-invariant definition of the phase difference $\Delta \chi$ between the two sides of the brane must involve the dual field $C^{\mu}$. Repeating the arguments that led to Eq. (6) we find

$$
\tilde{J}^{3}(\Delta \chi)=\tilde{J}_{\max }^{3} \sin (\Delta \chi) .
$$

We emphasize at this point that the presence of a current is independent of the detailed form of the Lagrangian of the system. It is a consequence only of our assumption that a condensate exists on either side of the brane [12].

We turn next to the gauge field localized on the brane. The Maxwell equations read

$$
\begin{gathered}
\partial_{\mu} F^{\mu \nu}=0, \\
\partial_{\mu} \tilde{F}^{\mu \nu}=\tilde{J}^{\nu} .
\end{gathered}
$$

In the second equation we have included the tunnelling magnetic current $\tilde{J}^{3}$. We must also take into account the constraints on the electromagnetic field arising from the presence of the dual superconducting phase in the bulk. The arguments we gave in the case of the standard Josephson junction can be repeated with the exchange of the role of electric and magnetic fields (Fig. 1).

The Maxwell equations can be solved easily in terms of the dual gauge field $C^{\mu}$ defined in Eq. (12). The nonvanishing components of the electromagnetic field are 


$$
E_{x}=-\frac{\partial C^{3}}{\partial y} E_{y}=\frac{\partial C^{3}}{\partial x} B_{z}=-\frac{\partial C^{3}}{\partial t},
$$

where we have assumed no $z$-dependence. The reasoning that led to Eq. (9) now gives

$$
\partial^{i} \partial_{i}(\Delta \chi)+g_{m} d \tilde{J}_{\max }^{3} \sin (\Delta \chi)=0 .
$$

For $\Delta \chi \ll 1$ we obtain

$$
\left[\partial^{i} \partial_{i}+\tilde{m}^{2}\right] \Delta \chi=0,
$$

with $\tilde{m}^{2}=g_{m} d \tilde{J}_{\max }^{3}$. The massive mode $\Delta \chi$ can be identified with the third component of the dual field: $\Delta \chi=$ $-g_{m} d C^{3}$.

In summary, the following picture emerges: $\mathrm{A}(2+1)-$ dimensional low-energy theory appears on the brane. It involves one physical degree of freedom that can be identified with the dual gauge field $C^{3}$. The components of the electromagnetic field $E_{x}, E_{y}, B_{z}$ are given by the simple expressions (17). The field $C^{3}$ is massive, with a mass $\tilde{m}$ that can be very small in units of the typical scale of the theory in the bulk. As a result, the electromagnetic field has a finite correlation length. This can be seen by simply taking $y, x, t$-derivatives of Eq. (19) and remembering that $\Delta \chi=-g_{m} d C^{3}$. Indirect experimental support of this picture comes from the observation of a Meissner effect in standard Josephson junctions.

We expect the above conclusions to remain valid in a theory that includes electric charges on the brane. As we mentioned earlier, the Josephson effect is an immediate consequence of the presence of charged condensates and does not depend on the details of the underlying theory [12]. Therefore, the complications encountered in constructing a consistent theory of electric and magnetic charges are not expected to lead to significant modifications of our arguments. We mention that, in a consistent theory, electric and magnetic charges must satisfy Dirac's quantization condition [16]

$$
g_{e} g_{m}=2 \pi n \text {. }
$$

An interesting solution of the sine-Gordon equation (18) is given by [8]

$$
\Delta \chi=2 \sin ^{-1} \operatorname{sech}\left[\tilde{m}\left(x-x_{0}\right)\right] .
$$

It corresponds to a defect localized near the line $x=x_{0}$. The electric field $E_{y}$ is nonzero near $x_{0}$ and vanishes at distances $\gtrsim \tilde{m}^{-1}$ away from it. The phase $\Delta \chi$ changes by $2 \pi$ as $x$ goes from $-\infty$ to $\infty$. It is easy to see that the defect carries unit electric flux $2 \pi / g_{m}=g_{e}$, for $n=1$ in Eq. (20). The energy per unit length of the defect is $\sim\left(\tilde{J}_{\max }^{3} / g_{m}^{3} d\right)^{1 / 2}$ [8]. Lines with larger electric flux correspond to solutions for which $\Delta \chi$ varies by multiples of $2 \pi$. It is natural to expect that flux-carrying lines of this type connect opposite electric charges on the brane.

There is a close similarity between the bulk and the brane of width $d$. On the brane the electromagnetic field is massive and defects exist that carry electric flux, similarly to the behavior in the bulk. The most consistent interpretation of the emerging $(2+1)$-dimensional theory is that it displays confinement with a linear potential, but with a typical scale much smaller than the scale characterizing the theory in the bulk. This is in agreement with the experimental studies of standard Josephson junctions. In that case the system behaves as if the superconducting properties extend over the whole structure including the barrier [8]. In a certain sense, this is caused by the electric condensate penetrating the barrier instead of ending abruptly at the surface. For the dual picture that we are considering, we expect the magnetic condensate to behave in an analogous way. The implication is that dual superconducting behavior, and therefore confinement, must be present inside the brane as well.

\section{Compact QED in $2+1$ dimensions}

The $U(1)$ gauge theory that emerges at low energies through the localization mechanism discussed in Sec. II is compact. This is a consequence of the fact that the $U(1)$ symmetry is embedded in the non-Abelian $S U(2)$ group. The low-energy theory has strong similarities with the Georgi-Glashow model [13] in $2+1$ dimensions. The unbroken $U(1)$ gauge symmetry in this model again results from the breaking of $S U(2)$. The theory contains instantons, which correspond to the monopoles of the $(3+1)$ dimensional theory when the Euclidean time and the third spatial dimension are interchanged. The smallest magnetic charge is $g_{m}=4 \pi / g$, where $g$ is the gauge coupling constant. The unit electric charge in the Georgi-Glashow model is $g_{e}=g / 2$, so that $g_{e}, g_{m}$ satisfy Eq. (20) with $n=1$.

Polyakov [9] has demonstrated that the presence of instantons destroys the Coulomb phase, moving the theory to a confining one. The sector with $n=1$ in Eq. (20) dominates the dynamics. The confinement scale is exponentially suppressed by the action of the instantons. This conclusion is in agreement with the physics of the Josephson junction. In the Josephson picture the confinement is a consequence of the tunnelling monopoles of the $(3+1)$-dimensional theory. It seems natural to identify them with the instantons of the $(2+1)$-dimensional theory. In the following section we shall make this relation concrete.

Before discussing the details of this connection, we point out some other striking similarities between the two pictures. In the $(2+1)$-dimensional theory, the confining dynamics is dominated by the presence of widely separated instantons. The relevant partition function is very similar to that of the Coulomb gas. In this work we are interested in the case of a large Higgs mass $\left(m_{H} \gg m_{W}\right.$ at the minimum $\Phi_{0}$ of the Higgs potential) [17]. The partition function of the $(2+1)$-dimensional theory can be cast in the form $[9,18]$ 
$Z \sim \int \mathcal{D} \tilde{\chi} \exp \left(-\frac{g^{2}}{32 \pi^{2}} \int d^{3} x\left[\partial^{\mu} \tilde{\chi} \partial_{\mu} \tilde{\chi}+2 \tilde{M}^{2} \cos (\tilde{\chi})\right]\right)$,

with $\tilde{M} \sim \exp \left(-S_{0}\right)$, where $S_{0}$ is the instanton action $S_{0}=$ $4 \pi \epsilon \Phi_{0} / g$, with $\epsilon \simeq 1.787$. The field $\tilde{\chi}$ is the dual photon and corresponds to the single physical degree of freedom of the $(2+1)$-dimensional gauge theory. Its equation of motion is given by (18), with $\widetilde{J}_{\max }^{3} \sim \exp \left(-S_{0}\right)$. It is clear that the field $\tilde{\chi}$ of the $(2+1)$-dimensional theory can be identified with the field $C^{3} \sim \Delta \chi$ in the picture of the Josephson junction.

It is obvious from the above that the physics of the localized gauge theory can be described equivalently in two different ways: (a) through the picture of the Josephson junction, with the tunnelling monopoles playing a major role; (b) in purely $(2+1)$-dimensional terms, through the compact $U(1)$ gauge theory, in which the instantons are the dominant entities. In the following, we demonstrate the equivalence of the two pictures employing topological arguments.

\section{DESCRIPTION IN TERMS OF TOPOLOGICAL ENTITIES}

We now wish to establish a connection between the long-distance confining dynamics of the $U(1)$ theory on the brane and the confinement in the $(2+1)$-dimensional $S U$ (2) Higgs theory. Qualitatively, it is clear that this connection should come from the fact that in both cases there is a $U(1)$ symmetry embedded in $S U(2)$ above a certain scale. However, the difficulty in applying the experience from the $(2+1)$-dimensional case arises because the $U(1)$ theory gets directly embedded in a $(3+1)$ dimensional $S U(2)$. This, although Higgsed on the brane, is never in a Higgs phase in the $(2+1)$-dimensional sense. In other words, the localization of the $(2+1)$-dimensional $U(1)$ is a result of the interplay between the bulk confinement and the brane Higgs effect. The two effects cannot be decoupled. There is no intermediate window of scales within which one could decouple the confining bulk physics, and in the same time ignore the brane Higgs effect, in such a way that the effective theory in this interval can be regarded as a $(2+1)$-dimensional $S U(2)$.

In order to circumvent this complication we shall employ topological arguments, which will allow us to trace the origin of the instantons in the effective low-energy theory on the brane in terms of higher-dimensional topological entities. This will allow us to bypass the full complicated confining dynamics in the bulk. The method that we shall discuss is useful more generally for visualizing the topological structure of theories in arbitrary dimensions in terms of topological entities of a higher-dimensional theory, even if the latter is a pure mathematical extrapolation.

The connection between the topological defects in a $(D+1)$-dimensional theory with the instantons in
$D$-dimensions is well known. The important point of this connection is that, by viewing the Euclidean time coordinate in $D$ dimensions as the $D$-th space coordinate in $D+$ 1 dimensions, the $D$-dimensional instanton solution becomes a topological defect in $D+1$ dimensions.

In the same time instantons in $D$ dimensions describe transitions between vacua with different topological winding numbers. The transition between different vacua in $D$ dimensions can be given an explicit topological meaning in terms of the motion of a soliton in $D+1$ dimensions as follows: Imagine that the spatial part of the $D$-dimensional theory is a $D-1$ sphere of radius $R$, embedded in a fictitious $D$-dimensional space. The fields of the lowerdimensional theory $\Phi(x)$, where $x$ denotes the worldvolume coordinates of the sphere, can be viewed as expectation values of higher-dimensional fields $\Phi(x, y)$ on the sphere $(y=R)$. A topological vacuum of the $D$-dimensional theory with nonzero winding number simply corresponds to a configuration in which a monopole is placed at the center of the sphere in $D$ space dimensions. The $D$-dimensional instanton driving the transition between different vacua corresponds to the motion of the monopole across the sphere in the $D+1$-dimensional picture. Changing the winding number in the first picture is equivalent to changing the monopole number enclosed by the sphere in the second picture. This connection becomes much clearer in the specific examples we consider in the following.

\section{A. Instantons in two dimensions as tunnelling vortices}

Consider the $(1+1)$-dimensional Abelian Higgs model

$$
S=\int d^{2} x\left\{-\frac{1}{4} F_{\mu \nu} F^{\mu \nu}+\left|\mathcal{D}_{\mu} \Phi\right|^{2}-\lambda\left(|\Phi|^{2}-v^{2}\right)^{2}\right\}
$$

where $\Phi \equiv \rho \exp (i \omega)$ is a complex scalar Higgs field. The minima of the potential form a circle with radius $\rho=v$. Let us imagine that the spatial dimension is also a compact circle of some radius $R$ much larger than the inverse Higgs mass. The possible vacua of this theory can be characterized by a nontrivial topological winding number

$$
n=\frac{1}{2 \pi} \int d \phi(\partial \omega / \partial \phi)=\frac{\omega(2 \pi)-\omega(0)}{2 \pi} .
$$

The explicit form of the Higgs field is $\Phi_{\text {vac }}=v \exp (i n \phi)$, with the coordinate $\phi$ changing from 0 to $2 \pi$ around the circle. The configuration of the gauge field corresponds to a pure gauge. Similar vacua exist also in the noncompact case, with the fields at the points $x=-\infty$ and $x=\infty$ differing by a large gauge transformation.

In this theory there are also instantons, that correspond to transitions between vacua with different $n$ [19]. If the spatial dimension is noncompact, an instanton is given by a vortex configuration [11] in the two-dimensional space that results from switching to imaginary time $t_{e}$. Its explicit 
form is

$$
\begin{gathered}
\Phi_{\mathrm{inst}}=f\left(r_{e}\right) \exp \left[\operatorname{in} \phi_{e}\right], \quad r_{e}^{2} \equiv x^{2}+t_{e}^{2}, \\
\phi_{e} \equiv \arctan \left(t_{e} / x\right) .
\end{gathered}
$$

Here $f(r)$ is the Nielsen-Olesen function that satisfies $f(0)=0, f(\infty)=v[11]$. The gauge field corresponds to a pure gauge.

For a compact spatial dimension the instanton transition can be visualized as follows: Imagine that the circle of radius $R$ on which our model lives is embedded in a twodimensional plane. The $(1+1)$-dimensional fields can be viewed as being determined by their higher-dimensional extensions evaluated on the circle. The vacua with winding number $n$ on the circle correspond to configurations in which there are $n$ vortices enclosed by the circle. This becomes obvious if we place a vortex with winding number $n$ at the center. This configuration on the circle is described by the first relation of (25) with the replacements $\phi_{e} \rightarrow \phi$, $r_{e} \rightarrow R$, where now $\phi$ is the angular coordinate. Obviously, the integral (24) is then equal to $n$. The instantons that change this winding number correspond to the
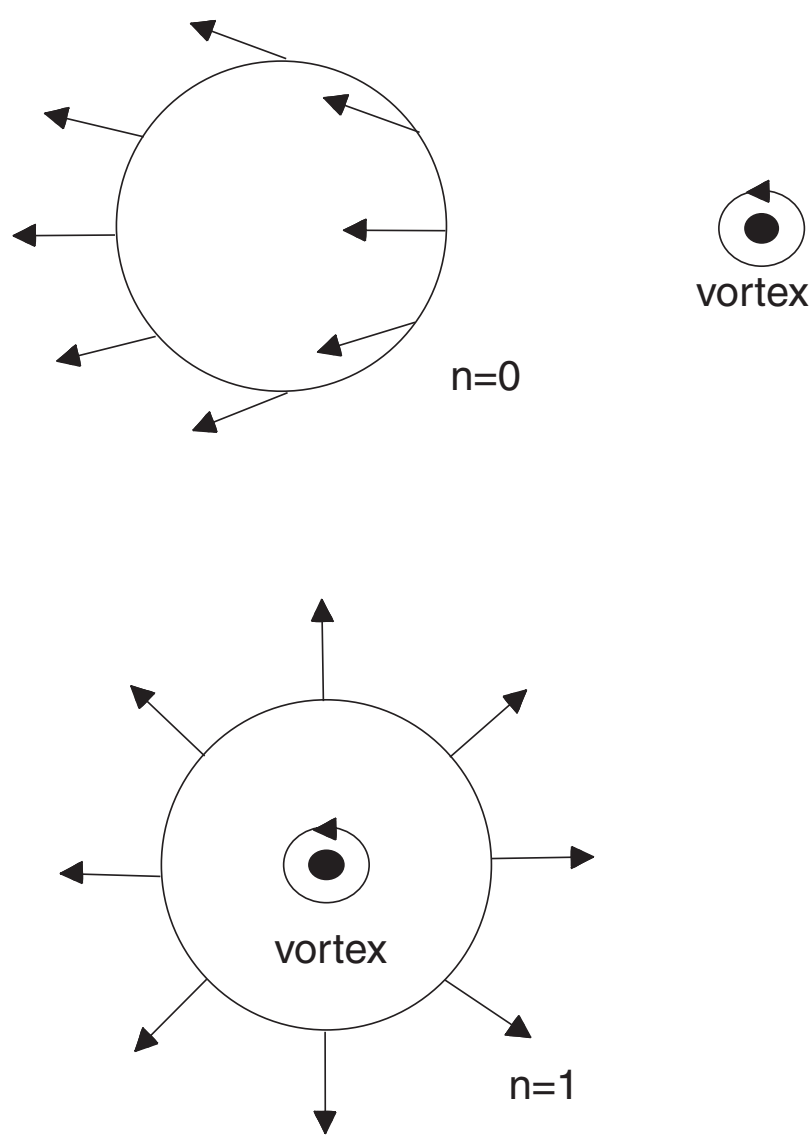

FIG. 2. A process that changes the winding number of the vacuum by one unit in the $(1+1)$-dimensional Abelian Higgs model. change of the vortex number inside the circle in the $(2+1)$-dimensional picture.

For example, the transition from a trivial vacuum to one with winding number $n$ is equivalent to a vortex with number $n$ entering the circle of radius $R$. This process is depicted in Fig. 2 for $n=1$. The black dot denotes a vortex, initially located outside the circle. The arrows indicate the orientation of the Higgs field in internal space. They are taken to have equal length, as the magnitude of the Higgs field is $v$ far from the vortex center. It is apparent that initially the vacuum winding number for the $(1+1)$ dimensional theory on the circle is zero. When the vortex moves inside the circle the winding number increases by one unit.

\section{B. Instantons in three dimensions as tunnelling monopoles}

The discussion of the previous subsection can be generalized to higher dimensions. Consider the $(2+1)$ dimensional $S U(2)$ Higgs model

$$
\begin{aligned}
S= & \int d^{3} x\left\{-\frac{1}{4} F_{\mu \nu}^{a} F_{a}^{\mu \nu}+\mathcal{D}_{\mu} \Phi^{a} \mathcal{D}^{\mu} \Phi_{a}\right. \\
& \left.-\lambda\left(\Phi^{a} \Phi_{a}-v^{2}\right)^{2}\right\},
\end{aligned}
$$

with the Higgs field in the adjoint representation $a=1,2$, 3 . There exist various vacua that correspond to topologically nontrivial configurations. If the two-dimensional space is noncompact, the simplest such configuration is given by

$$
\Phi=v(\cos \phi \sin \theta(\rho), \sin \phi \sin \theta(\rho), \cos \theta(\rho)),
$$

in polar coordinates $\phi, \rho$. The function $\theta(\rho)$ interpolates between 0 and $\pi$ as $\rho$ goes from 0 to $\infty$. The corresponding topological number is

$$
n=\frac{1}{8 \pi v^{3}} \int d^{2} x \epsilon_{a b c} \epsilon^{0 \mu \nu} \partial_{\mu} \Phi^{a} \partial_{\nu} \Phi^{b} \Phi^{c}=1 .
$$

The two-dimensional space can be compactified to a twosphere of radius $R$ by identifying the points at infinity ( $\rho \rightarrow$ $\infty)$. We can imagine this sphere being embedded in a fictitious three-dimensional space. The configuration of Eq. (26) would originate from a monopole located at the center of the sphere. The $(2+1)$-dimensional instanton then would describe a process that moves a monopole through the two-sphere. During this process the winding number (28) changes by one unit. This demonstrates our main point: The $(2+1)$-dimensional instanton can be understood as a process that takes the monopole across the space from an imaginary third dimension.

The application to the problem of gauge-field localization is straightforward. We consider a $(3+1)$-dimensional $S U(2)$ model with an adjoint Higgs 


$$
S=\int d^{4} x\left\{-\frac{1}{4} F_{\mu \nu}^{a} F_{a}^{\mu \nu}+\mathcal{D}_{\mu} \Phi^{a} \mathcal{D}^{\mu} \Phi_{a}-V(\Phi)\right\} .
$$

The potential $V(\Phi)$ has two degenerate minima, one with $\Phi=0$ and the other with $\Phi^{a} \Phi_{a}=M^{2}$. An example of such a potential is given by (2). The first vacuum is confining at some scale $\Lambda$ and there is a mass gap. There are no massless states there. In the second vacuum $S U(2)$ is Higgsed down to $U(1)$ and there is a massless photon. The two phases can coexist, separated by walls of thickness $\sim M^{-1}$ and tension $\sim M^{3}$. We assume that $M \gg \Lambda$. We consider a layer of the $\Phi \neq 0$ phase sandwiched between the $\Phi=0$ phases. We assume that the thickness of the layer is $d \gg M^{-1}$. The walls then have an exponentially suppressed interaction and can be considered to be static during the time scales of interest.

The important point is that configurations in this model, just like the ones in the truly $(2+1)$-dimensional Higgs model, can be characterized by a topological winding number. An example is given by

$$
\Phi=f(z)(\cos \phi \sin \theta(\rho), \sin \phi \sin \theta(\rho), \cos \theta(\rho)),
$$

where $z$ is the coordinate perpendicular to the layer, and $f(z)$ describes the parallel wall and antiwall: It vanishes for $z \rightarrow \infty$ and $-\infty$ and takes the value $M$ within a layer of width $d$ around $z=0$. The corresponding winding number is

$$
n=\frac{1}{8 \pi v^{3}} \int d^{3} x \epsilon_{a b c} \epsilon^{30 \mu \nu} \partial_{\mu} \Phi^{a} \partial_{\nu} \Phi^{b} \Phi^{c} .
$$

This winding number can be changed by taking a monopole through the layer. The instanton of the $(2+1)$ dimensional theory describes this process. As the mass of the monopole in the layer is given by the expectation value of $\Phi=M$, in the limit $M \gg \Lambda \gg d^{-1}$ the process must be exponentially suppressed by the factor $\exp (-M d)$. The reason is that $M d$ sets the barrier that the monopoles have to tunnel through. In the opposite case $M \sim d^{-1} \gg$ $\Lambda$ the suppression factor should be $\exp (-M \Lambda)$.

\section{Form of the flux tubes}

As we discussed in previous sections, the resulting lowenergy theory is strongly confining in the bulk, while it displays weak confinement of electric charges on the brane. In Fig. 3 we depict the different form of a flux tube in the bulk and on the brane. The configuration of Fig. 3 is generated by an electric charge located in the bulk and another one on the brane. The electric flux lines form a tight tube in the bulk, of width $\sim 1 / \Lambda$, where $\Lambda$ is the bulk confinement scale. On the brane the confinement scale is exponentially suppressed, so that the width of the flux tube increases dramatically. On the other hand, no flux lines escape to infinity. They all start and finish on the charges.

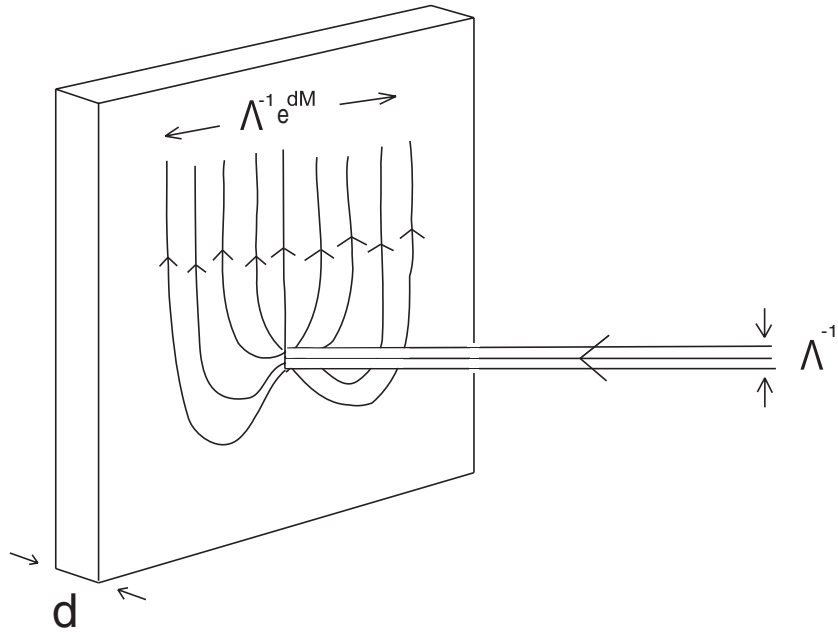

FIG. 3. Spreading of a flux tube ("open string") on a layer ("D-brane").

This configuration has a very strong similarity to an open string with its end attached on a $D$-brane in string theory.

\section{FOUR DIMENSIONS AND PHENOMENOLOGY}

\section{A. The case of $(3+1)$-dimensional electrodynamics}

As we have seen, the effective $(2+1)$-dimensional electrodynamics becomes confining at exponentially large distances. This happens because the UV-completing $S U(2)$ physics does not decouple entirely, but leaves an imprint on the IR physics. This imprint, although parametrically negligible at short length scales, becomes dominant at exponentially large distances and makes the $U(1)$ theory confining.

The natural question to ask is whether this effect is exceptional to $2+1$ dimensions, or whether it also takes place for the localization of the photon onto a $(3+1)$ dimensional brane in higher dimensions. The standard intuition in $3+1$ dimensions tells us that the latter scenario is impossible, because, from what is known, the IR behavior of the pure $U(1)$-theory in $3+1$ dimensions is insensitive to the UV completion. However, as we are dealing with an extra-dimensional completion of the theory, the story may be much less straightforward.

There are two possible cases:

(i) The first possibility is that the theory below a certain scale reduces to simple $U(1)$ electrodynamics with a massless photon with two polarizations, and no extra light degrees of freedom. In this case, we can make a strong argument that the $(3+1)$-dimensional localization is very different from the analogous one in $2+1$ dimensions. Also, confinement can take place in the latter case but not in the former.

The argument is based on counting and matching the number of degrees of freedom for the massless $U(1)$ theory and the massive confining one. Consider the 
$(2+1)$-dimensional case first. As explained above, the theory includes two mass scales. The first one is the scale $d^{-1}$ (width of the brane) below which the effective low-energy theory is that of a massless $(2+1)$-dimensional photon, with one propagating degree of freedom. The second is the scale $\exp (-M d)$, below which the theory confines and becomes one of the composite massive glueballs, with the lowest one being a scalar. The crucial point is that the degrees of freedom needed for describing the two theories can match. Both a massive scalar glueball and a massless vector field carry the same number of propagating physical degrees of freedom. In other words, the massless $U(1)$ theory can smoothly flow into the theory of a massive scalar glueball, without any need of extra degrees of freedom. Notice that this would be impossible if the mass of the photon were of the Higgs or Proca type, since in such a case an extra massless degree of freedom (a Goldstone-Stuickelberg field) is necessary, that would become the longitudinal polarization of the massive photon.

In $3+1$ dimensions such a matching of modes is impossible. The massless photon carries 2 degrees of freedom, and this number cannot be matched with the number of degrees of freedom in any massive representation of the Lorentz group. Thus, the massless $(3+1)$-dimensional $U(1)$ theory cannot smoothly flow to a theory of massive glueballs in the far infrared without acquiring some extra degrees of freedom from outside.

(ii) The second possibility is that the additional light degrees of freedom needed for the infrared confinement are provided by the UV-completing extradimensional physics. Even though we do not know of a specific implementation of such a scenario, we cannot rule out this possibility, especially if the extra dimensions have infinite volume and no mass gap.

\section{B. Phenomenological bound on the photon confinement scale}

The most obvious experimental signature for the scenario we considered is that electrodynamics would become confining at exponentially large distances. It is important to point out that the phenomenological bounds on such a confinement scale would be much more severe than the bounds on a photon mass of the Higgs or Proca type. Interestingly, this bound follows from the existence of a long-range galactic magnetic field. This would be screened by the magnetic condensate if the confinement scale of the photon were larger than the inverse galactic size, that is $10^{-27} \mathrm{eV}$. The bound on the Higgs-type mass of the photon is much milder, only about $10^{-16} \mathrm{eV}$ [20], because such a mass cannot screen the galactic magnetic field, but only the electric one, which is absent anyway.

\section{CONCLUSIONS}

This paper was devoted to the investigation of the duality between the gauge-field localization mechanism of [3] and the physics of the Josephson junction. It was suggested in [6] that, if the low-energy duality is complete, the $(2+1)$-dimensional electrodynamics must become confining at exponentially large distances. By invoking topological methods we identified explicitly the sources of this confinement. They are the instantons that correspond to the tunneling across the brane of the monopoles that are condensed in the bulk. The resulting current is completely analogous to the Josephson current.

The IR dynamics of the theory that arises through the localization mechanism of [3] seems sensitive to the UV completion. In particular, complete decoupling of the UV does not take place, as there is always a tunnelling current that is crucial for the IR behavior. This current flows because of the presence of magnetic condensates of the $(3+1)$-dimensional $S U(2)$ theory on either side of the brane. It has, therefore, a $(3+1)$-dimensional character. On the other hand, the effect is exponentially suppressed. The long-distance confining dynamics of the $U(1)$ theory on the brane is very similar to the confinement in the $(2+1)$-dimensional $S U(2)$ Higgs theory with the symmetry broken down to $U(1)$. In both cases there is a $U(1)$ symmetry embedded in $S U(2)$ above a certain scale. The long-distance dynamics reflects this embedding, which results in the presence of tunnelling monopoles in the first case and instantons in the second.

The most exciting aspect of the scenario we considered is that it has experimental low-energy implications for a nongravitational sector. It demonstrates that the presence of extra dimensions can be unveiled much below the energies at which particles are released in the bulk. We discussed the possible generalization of the effect for higher dimensions and the phenomenological bound on the infrared confinement of the photon. Interestingly, this bound is much more severe than the one on a photon mass of the Higgs or Proca type. It follows from the existence of the galactic magnetic field, which cannot constrain a Higgs-type photon mass very efficiently.

\section{ACKNOWLEDGMENTS}

The work of G. D. is supported by the David and Lucile Packard Foundation and the NSF Grant No. PHY-0245068. The work of N.T. is supported by the research program "Pythagoras II" (Grant No. 70-03-7992) of the Greek Ministry of National Education, partially funded by the European Union, the research program Kapodistrias of the University of Athens, and by the European Commission under the Research and Training Network Contract No. MRTN-CT-2004-503369. N. T. would like to thank the CERN Theory Institute for its hospitality during the time that this work was carried out. 
[1] R. Jackiw and C. Rebbi, Phys. Rev. D 13, 3398 (1976); E. J. Weinberg, Phys. Rev. D 24, 2669 (1981).

[2] J. Polchinski, String Theory (Cambridge University Press, Cambridge, England, 1998).

[3] G. R. Dvali and M. A. Shifman, Phys. Lett. B 396, 64 (1997); 407, 452(E) (1997).

[4] G. Dvali and A. Vilenkin, Phys. Rev. D 67, 046002 (2003).

[5] E. Witten, Nucl. Phys. B507, 658 (1997); G. R. Dvali and Z. Kakushadze, Nucl. Phys. B537, 297 (1999); M. A. Shifman, Phys. Rev. D 59, 021501 (1998); G. R. Dvali, G. Gabadadze, and Z. Kakushadze, Nucl. Phys. B562, 158 (1999).

[6] N. Tetradis, Phys. Lett. B 479, 265 (2000).

[7] S. Mandelstam, Phys. Rep. 23, 245 (1976); G.'t Hooft, Nucl. Phys. B190, 455 (1981).

[8] For reviews with references to original papers, see B. D. Josephson, in Superconductivity, edited by R. D. Parks (Marcel Decker, New York, 1969); A. Barone and G. Paternò, Physics and Applications of the Josephson Effect (John Wiley \& Sons, New York, 1982).
[9] A. M. Polyakov, Nucl. Phys. B120, 429 (1977).

[10] E. Adelberger, G. Dvali, and A. Gruzinov, Phys. Rev. Lett. 98, 010402 (2007).

[11] H. B. Nielsen and P. Olesen, Nucl. Phys. B61, 45 (1973).

[12] S. Weinberg, Prog. Theor. Phys. Suppl. 86, 43 (1986).

[13] H. Georgi and S. L. Glashow, Phys. Rev. Lett. 28, 1494 (1972).

[14] D. Zwanziger, Phys. Rev. D 3, 880 (1971).

[15] M. Blagojevic and P. Senjanovic, Phys. Rep. 157, 233 (1988).

[16] P. A. M. Dirac, Proc. R. Soc. A 133, 60 (1931).

[17] For $m_{H} \gg m_{W}$ the boundaries of the brane are very sharp, in agreement with our assumptions throughout the paper.

[18] K. Dietz and T. Filk, Nucl. Phys. B164, 536 (1980).

[19] R. Rajaraman, Solitons and Instantons (North-Holland, Amsterdam, 1987).

[20] W. M. Yao et al. (Particle Data Group), J. Phys. G 33, 1 (2006). 